\title{
Hale-Bopp Observations with Hubble and IUE Surprise Astronomers
}

Hubble Space Telescope News, NASA

Completing an unprecedented yearlong study of Comet Hale-Bopp using two NASA observatories, the Hubble Space

Telescope and the International

Ultraviolet Explorer (IUE), astronomers report that they are surprised to find that the different ices in the nucleus seen to be isolated from each other. They also report seeing unexpectedly brief and intense bursts of activity from the nucleus during the monitoring period. The Hubble observations suggest that the nucleus is huge, $19-25$ miles $(30-40 \mathrm{~km})$ across. The key results are described in the following. place, with "vents" being turned on and off as new patches of icy material are rotated into sunlight for the first time.

\section{A Complex, Mottled Nucleus}

To their surprise, astronomers found that water ice sublimates (turns directly from the frozen solid into a gas) at a different rate than the trace ices, implying that those components are not contained within the water on the comet. This conclusion is further supported by Hubble data showing that the rate at which dust left the

\section{A Monstrous Nucleus}

By studying Hubble Space Telescope images the astronomers have estimated that Hale-Bopp's nucleus may be about 19-25 miles $(30-40 \mathrm{~km})$ in diameter. The average comet is thought to have a nucleus of about 3 miles $(5 \mathrm{~km})$ in diameter, even smaller. The comet or asteroid that struck the Earth 65 million years ago, possibly causing the extinction of the dinosaurs, was probably about $6-9$ miles $(10-15 \mathrm{~km})$ across.

Because Hale-Bopp was unusually bright when it was still a great distance away, well outside the orbit of Jupiter, it has given scientists their best view ever of the changes in a comet's nucleus as it gets closer to, and is progressively heated by, the sun. Those changes, in turn, provide information about the composition and structure of comets, which are believed to be remnants from the formation of the solar system, about 4.6 billion years ago. Learning more about comets can provide important information about the materials and processes that formed the solar system.

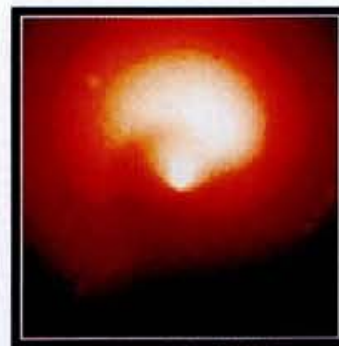

September 26, 1995

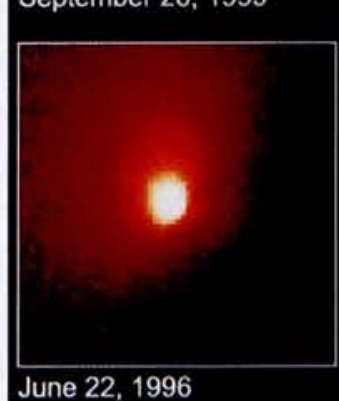

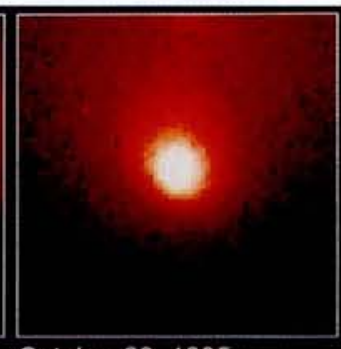

October 23, 1995

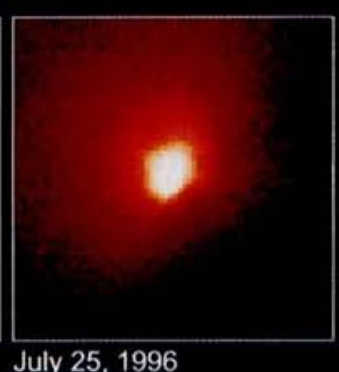

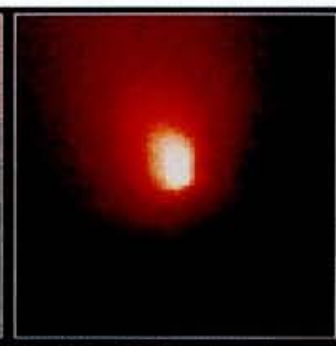

April 7, 1996

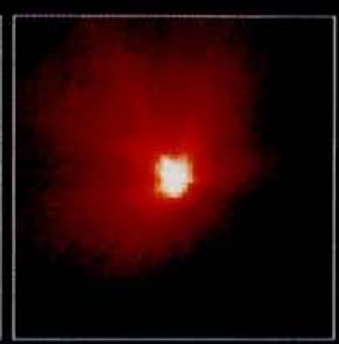

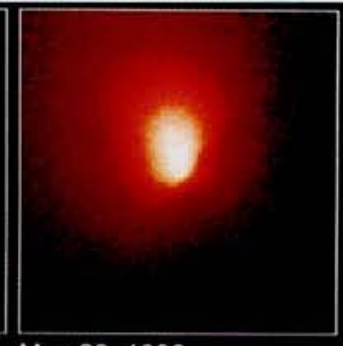

May 20, 1996

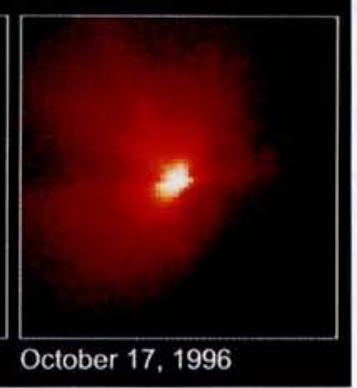

Fig. 1. Comet Hale-Bopp, Hubble space telescope, wide field planetary caamera 2
Violent Eruptions on the Comet's Surface

During the course of long-term observations, which began in August 1995, astronomers unexpectedly caught the comet going through a sudden brief outburst, where, in little more than an hour the amount of dust being spewed from the nucleus increased at least eight-fold. It appears that the surface of Hale-Bopp's nucleus must be an incredibly dynamic nucleus was very different than the sublimation rate of water. This result is contrary to previous models for a comet's nucleus which suggest that the trace components, such as carbon disulphide ice, are contained inside the most abundant ice on the comet, frozen water. As water sublimates, the trace components and dust should be released at similar rates, but this is not what Hubble observed.
The Space Telescope Science Institute is operated by the Association of Universities for Research in Astronomy, Inc. (AURA), for NASA, under contract with the Goddard Space Flight Center, Greenbelt, MD. The Hubble Space telescope is a project of international cooperation between NASA and the European Space Agency (ESA). 